\title{
The role of part-whole information in reasoning about relative size
}

\author{
KEVIN M. SAILOR \\ Lehman College, City University of New York, New York, New York \\ and \\ EDWARD J. SHOBEN \\ University of Illinois, Champaign, Illinois
}

\begin{abstract}
Models of comparative judgment have assumed that relative magnitude is computed from knowledge about absolute magnitude rather than retrieved directly. In Experiment 1, participants verified the relative size of part-whole pairs (e.g., tree-leaf) and unrelated controls (e.g., tree-penny). The symbolic distance effect was much smaller for part-whole pairs than for unrelated controls. In two subsequent experiments, participants determined either which of two objects was closer in size to a third object or which of two pairs had a greater difference in the size of its constituents. In contrast to the paired comparison task in Experiment 1, judgments of part-whole items were more sensitive to the influence of symbolic distance than were unrelated controls. The fact that the part-whole relation attenuates the effects of symbolic distance in a paired comparison task but not in tasks that require an explicit comparison of size differences suggests that the part-whole relation provides a source of information about relative magnitude that does not depend on knowledge about absolute magnitude.
\end{abstract}

Much of our general world knowledge is knowledge about objects and their attributes. For example, the fact that coffee is darker than milk, that monkeys are smarter than dogs, or that ordering dinner requires much less time than calculating one's taxes can serve to distinguish each of these objects or events. An important topic in research on semantic memory is how people represent knowledge about the properties and attributes of objects and events and then use this knowledge to make these relational judgments.

These issues have been the primary focus of research on relational or comparative judgments (Banks, 1977; Holyoak, 1978; Holyoak, Dumais, \& Moyer, 1979; Shoben, Çech, Schwanenflugel, \& Sailor, 1989). In particular, the goal of this research is to determine how people make comparisons between objects or events along some dimension or property. For example, researchers have examined how people decide which of two objects is larger (Moyer, 1973), which of two animals is fiercer (Holyoak \& Mah, 1981), and which of two events occurs earlier (Nottenburg \& Shoben, 1980).

The most robust finding in comparative judgment is the symbolic distance effect. This effect is the finding that the

Experiment 1 partially fulfilled the requirements for K.M.S.'s doctoral degree at the University of Illinois. This research was supported in part by Grant S06GM08225 from NIH to Lehman College, and in part by Grant BN586-08215 from the National Science Foundation to E.J.S. We thank Michael Coles, Don Dulany, Gordon Logan, and Brian Ross for helpful comments throughout this research. Correspondence should be sent to K. M. Sailor, Department of Psychology, Lehman College, Bronx, NY 10468-1589 (e-mail: ksailor@alpha.lehman.cuny.edu). difficulty of determining the relative magnitude of two objects decreases as the difference in their magnitudes increases. For example, it is easier to decide that a horse is larger than a cat than it is to decide that a horse is larger than a donkey. The symbolic distance effect is robust and is obtained across many diverse dimensions (Audley \& Wallis, 1964; Friedman, 1978; Holyoak \& Mah, 1981; Holyoak \& Walker, 1976; Kerst \& Howard, 1977; Maki, 1981; Nottenburg \& Shoben, 1980).

The significance of this finding is that it suggests that comparative judgments are performed by comparing the stored absolute magnitudes of objects. If the ordering of any pair of items on a given dimension is explicitly represented as a stored proposition (e.g., "elephants are larger than fleas" or "elephants are larger than rhinos") and relative magnitude is determined by retrieving the appropriate proposition, then decision time would be a function of the availability of a proposition rather than a function of differences in absolute magnitude. In contrast, the fact that the difference in absolute magnitude between two objects is strongly correlated with decision difficulty implies that relative magnitude is calculated from these differences in absolute magnitude.

Acceptance of this view that comparative judgments are performed by comparing the stored magnitudes of objects has been remarkably uniform. In addition to the finding of a symbolic distance effect, researchers have generally believed that the memory demands of explicitly representing relative magnitude were implausible because the number of all possible pairwise combinations of even a relatively modest set of items is quite large. Together, these facts have led very few theorists to question the generality 
of the claim that relative magnitude is computed from stored absolute magnitude information, even though they have advanced rather diverse proposals about processing assumptions and representational format (Banks, 1977; Holyoak, 1978; Marks, 1972; Paivio, 1975).

There are, however, several reasons to be skeptical of the implicit claim of these models that relative magnitude is always computed from knowledge about the absolute magnitude of individual objects. First, there is considerable evidence that the category membership of items can play a role in judgments of relative magnitude. There are a number of studies (Kosslyn, Murphy, Bemesderfer, \& Feinstein, 1977; Maki, 1981; Pliske \& Smith, 1979; Sailor \& Shoben, 1993, 1996) in which manipulating the category membership of the terms in a linear order either eliminated or greatly attenuated the effect of symbolic distance. For example, Maki (1981) had participants judge which of two cities was further east or west. When the two cities were located in different states, no symbolic distance effect was obtained, but when two cities were in the same state, the usual distance effect occurred. These results have been interpreted to indicate that participants determined relative magnitude by using category membership rather than absolute magnitude on a substantial proportion of the trials in which the items belonged to different categories.

Second, judgments of associated pairs suggest that more general exceptions to the influence of symbolic distance may exist. Holyoak et al. (1979) contrasted judgments of relative magnitude for associated pairs (e.g., needle-haystack) and unassociated pairs (e.g., coinbarn). In addition to finding that judgments were generally faster for associated than for unassociated pairs, the authors found that this effect of association was much greater for pairs that were similar in magnitude (i.e., low symbolic distance). Thus, the effect of distance was more pronounced on unassociated pairs. This attenuation of the distance effect is exactly what one would expect to observe if the relative magnitude of highly associated pairs is explicitly represented in memory as a consequence of their frequent appearance in the same context.

According to this account, a significant portion of judgments about associated pairs could be made by retrieving directly the relative magnitude of a pair rather than by computing its relative magnitude from differences in the absolute magnitudes of the two objects. The overall symbolic distance effect was smaller for associated pairs than for unassociated pairs because symbolic distance did not affect the duration of these trials on which relative magnitude was retrieved but did affect the duration of most if not all of the judgments of unassociated pairs because relative magnitude had to be computed for these pairs. Moreover, the general advantage for associated pairs observed by Holyoak et al. (1979) can be explained by the assumption that it is frequently faster to retrieve relative magnitude than it is to compute relative magnitude. This explanation is a significant departure from most accounts of the judgment process because it assumes that it is possible to retrieve the relation between two items di- rectly in addition to computing this relation from their individual magnitudes. It is an important departure because it proposes that strictly ordinal information about a pair may play a role in comparative judgments even under circumstances in which it is possible to document a symbolic distance effect. Thus, this model implies that a symbolic distance effect is not prima facie evidence that comparative judgments are always computed from absolute magnitude because the use of ordinal information will reduce rather than eliminate the symbolic distance effect in most cases. We will refer to the claim that there are additional processes by which explicitly represented relative magnitude is retrieved on some occasions as the direct retrieval hypothesis and we will refer to models that incorporate this hypothesis as two-process models.

Unfortunately, the importance of the latter result is somewhat qualified because an alternative explanation of these findings can be advanced within the framework of strictly computational models. As Holyoak et al. (1979) noted, association frequently facilitates the use of information from semantic memory (Glass, Holyoak, \& O'Dell, 1974; Holyoak, Glass, \& Mah, 1976; Shoben, 1976). In fact, the ubiquity of this effect has been sufficient to motivate the incorporation of a general spreading activation mechanism into a number of influential memory models whose function is to improve access to information that is related to recently accessed information (Anderson, 1983; Collins \& Loftus, 1975). Within the framework of a spreading activation account, the presence of the item grapefruit in the pair grapefruit-orange could be expected to facilitate retrieval of information about oranges because this information might be partially activated by grapefruit.

If one assumes that the retrieval of size information is facilitated by association, then standard computational models can be revised to handle these effects of association. For example, Banks's (1977) discrete code model proposes that discrete magnitude codes are generated for objects during a search of memory and that relative magnitude judgments involve a comparison of these discrete codes. This model explains the symbolic distance effect by assuming that identical magnitude codes are more likely to be generated for items with similar magnitude than items with very different magnitudes. If identical codes are generated, additional attempts must be made to generate different magnitude codes before a decision can be made. Thus, more processing is typically required to generate magnitude codes that can be discriminated (e.g., large vs. small) when the magnitudes of two items are similar.

Banks's (1977) basic model can be modified by assuming that code generation for one concept is facilitated by an attempt to retrieve magnitude information for a second, related, concept. If several attempts are required to generate different codes for a close pair whose members have similar magnitudes, the cumulative effect of the association would be substantial because each attempt would take less time for a related pair than for an unrelated pair. On the other hand, the cumulative effect of as- 
sociation on far pairs whose members are of very different magnitudes would be much less significant because a single attempt might be required to generate different codes. Thus, association attenuates the effect of symbolic distance because close pairs typically require more processing than far pairs and differences in the rate of code generation should be magnified as the amount of processing increases.

Similar accounts can be devised for any model that assumes that symbolic distance increases reaction time (RT) because it increases the amount of processing that must be done to compute relative magnitude from knowledge about absolute magnitude that has been retrieved from memory. For instance, a variety of sequential sampling or evidence accrual models have been proposed (Link, 1990; Petrusic, 1992) to describe comparative judgments. These models predict the symbolic distance effect by assuming that the probability of sampling positive evidence increases as the difference in the magnitudes of two objects increases. This increased probability reduces RT because a set criterion can be reached in fewer iterations or samples. If one assumes that semantic association reduces the time required to take each sample, then an interaction between symbolic distance and semantic association arises because the cumulative effect of this reduction in the time required for each sample is much greater when the number of iterations required to reach a response criterion increases. For example, if low symbolic distance pairs require 10 more iterations than high symbolic distance pairs, then a change in the rate of sampling from 10 samples per second for unassociated pairs to 20 samples per second for associated pairs will mean that the distance effect for unassociated pairs is $1 \mathrm{sec}$ and $.5 \mathrm{sec}$ for associated pairs. We will call the assumption that association speeds the computation of relative magnitude from absolute magnitude the facilitation hypothesis.

The viability of the facilitation hypothesis has important implications for current models of comparative judgment. If it is correct, standard models will require only minor modifications to explain the effects of semantic association. However, if it can be demonstrated that the facilitation hypothesis is an inadequate account of the effects of semantic association, then much more significant revisions of these models will be required. The logic of the current experiments is to find a set of items for which either the facilitation or direct retrieval hypotheses might hold and conduct a series of tests that contrast the predictions of each of these accounts.

These tests contrast performance on associated pairs with performance on unrelated pairs in two tasks in which participants were required either to determine which of two alternatives was closer in size to a third or to decide which of two sets of pairs of items was farther apart in size. They differ from a standard comparative judgment task in that they require a direct evaluation of the differences in the magnitudes of two items rather than an evaluation of their ordering on a dimension. For example, one cannot determine that a horse is closer in size to a rhino than it is to a mouse by knowing that a horse is smaller than a rhino but larger than a mouse. Instead, one must determine the difference in the sizes of a rhino and a horse and compare this difference to the difference in the sizes of a horse and a mouse. This feature of these tasks means that strictly ordinal information about a pair cannot be used to make an accurate judgment. According to the facilitation hypothesis, performance in these tasks should be quite similar to performance in a standard comparative judgment task. On the other hand, the direct retrieval hypothesis predicts that little benefit should exist for associated pairs because knowledge about a pair's ordering should not help to solve these tasks.

\section{EXPERIMENT 1}

In Experiment 1, comparative judgments of the sizes of items that include a part-whole relation were compared to judgments of items that did not contain a partwhole relation and thus were unrelated. These materials were chosen because objects and their parts are generally highly associated and the difference between a part and its whole is obviously correlated with relative magnitude because objects are always larger than their parts. In fact, some linguists have argued that the basis for judging that any object " $x$ " is smaller than a second object " $y$ " is the understanding that $x$ could be part of $y$ or that $x$ could be contained in y (Wierzbicka, 1972). On this basis, we hypothesized that the part-whole relation should attenuate the effect of symbolic distance in a comparative judgment task. This prediction was tested by presenting participants with pairs of objects that varied in symbolic distance, and in half of the cases bore a part-whole relation to each other.

\section{Method}

Materials. Eighty-eight pairs of words were drawn from a set of 279 words. In two previous norming experiments, one group of participants rated the size of 279 items on a 20 -point scale and a second group of participants rated the part-whole relatedness on a 7-point scale of 175 pairs of these same words. From this pool, 22 partwhole pairs with a relatively high symbolic distance (e.g., car-engine) were selected and 22 part-whole pairs with relatively low symbolic distances (e.g., hand-finger) were selected. Symbolic distance was calculated by subtracting the rated magnitude of the smaller object from the rated magnitude of the larger object. The mean symbolic distance was 4.98 scalar units for the high symbolic condition and 1.16 scalar units for the low symbolic distance condition. The mean rated goodness of the part-whole relation was roughly comparable for the high symbolic distance items (5.29) and the low symbolic distance items (5.34). A comparable set of high and low symbolic distance pairs that were unrelated were formed by re-pairing the members of the part-whole pairs to form 44 unrelated pairs. Thus, the same words served to form the stimuli in both the part-whole and the unrelated conditions for a given level of symbolic distance.

Design. The experimental items used in this study varied both in symbolic distance and in terms of whether or not the items formed a part-whole pair. Sample stimulus pairs are shown in Table 1.

Procedure. Each pair was presented six times during the course of the experiment. Pairs were ordered to form a true statement on $50 \%$ of the trials (larger item on the left) and a false statement on 
Table 1

Examples of Stimulus Pairs, Magnitude Difference, and Rated Goodness of Part-Whole Relation in Experiment 1

\begin{tabular}{ccc}
\hline \multicolumn{1}{c}{ Sample Pairs } & $\begin{array}{l}\text { Magnitude } \\
\text { Difference }\end{array}$ & $\begin{array}{c}\text { Part-Whole } \\
\text { Relatedness }\end{array}$ \\
\hline $\begin{array}{l}\text { High Symbolic Distance } \\
\text { car headlight }\end{array}$ & 4.98 & 5.29 \\
$\begin{array}{l}\text { Low Symbolic Distance } \\
\text { shirt collar }\end{array}$ & 1.16 & 5.34 \\
\hline
\end{tabular}

the remaining trials. The resulting 528 trials were divided into three blocks of 176 trials. The ordering of stimuli was randomly determined for each participant. Pairs were presented on IBM XTs equipped with EGA monitors. Each trial began with a ready signal (the word ready) that remained on the screen until the participant pressed the space bar, at which point the test pair appeared on the screen in uppercase letters. Participants were informed that word pairs constituted "abbreviated sentences" of the form " $\mathrm{X}$ is larger than $Y$ " where $X$ was the first word and $Y$ the second. This arrangement meant that when the left-hand word was the larger object the "sentence" was true, and when the left-hand word was the smaller object the sentence was false. Participants indicated that the trial was true by pressing the key labeled " 9 " with their right hand or that it was false by pressing the key labeled " 5 " with their left hand. RT was measured from the onset of the stimulus pair. If the participant made an incorrect response, the message "WRONG ANSWER" appeared for $4 \mathrm{sec}$.

Participants were given detailed feedback about their performance at the end of each block. They were informed about both their accuracy and their mean response latency. Participants were strongly encouraged to respond accurately on at least $95 \%$ of their trials and to respond as quickly as possible while maintaining this level of accuracy. Prior to the advent of the experimental trials, participants performed eight practice trials with materials not among the test stimuli.

Participants. The participants were 21 undergraduates at the University of Illinois who participated in partial fulfillment of a course requirement. All were right handed and native speakers of English They participated in semiprivate cubicles in groups of 3 or fewer.

\section{Results}

The results appear in Table 2. Mean RTs and error rates are given separately for each level of symbolic distance and relatedness. Overall error rates were moderately low (7\%) and correlated positively with RT (.67). Any RT more than $3.5 S D$ s from the mean RT of a participant was excluded from further analysis.

Consistent with previous findings that contrasted the effects of symbolic distance on unrelated pairs with semantically associated pairs (Holyoak et al., 1979), there was a strong interaction between symbolic distance and relatedness. As Table 2 indicates, the symbolic distance effect was $364 \mathrm{msec}$ for unrelated pairs but only $162 \mathrm{msec}$ for part-whole pairs. This interaction between relatedness and symbolic distance was highly reliable $[F(1,20)=$ $\left.113.8, M S_{\mathrm{e}}=1,872, p<.001\right]$.

In addition to this strong interaction between relatedness and symbolic distance, there were main effects of both relatedness and symbolic distance: Part-whole pairs were $263 \mathrm{msec}$ faster than unrelated pairs $[F(1,20)=$ $\left.1153.6, M S_{\mathrm{e}}=9,454, p<.0001\right]$, and high symbolic dis- tance pairs were $238 \mathrm{msec}$ faster than low symbolic distance pairs $\left[F(1,20)=284.8, M S_{\mathrm{e}}=4,183, p<.001\right]$. Even though relatedness and distance interacted strongly, relatedness still speeded judgment for high symbolic distance pairs $\left[F(1,20)=66.6, M S_{\mathrm{e}}=4,150, p<.001\right]$ and distance still speeded judgments of part-whole pairs $\left[F(1,20)=109.8, M S_{\mathrm{e}}=1,806, p<.001\right]$. Thus, symbolic distance and relatedness generally speeded processing, but the effects of relatedness were more pronounced for low symbolic distance pairs and the effects of symbolic distance were more pronounced for unrelated pairs.

In general, the error data support the results of the RT analyses. The accuracy of part-whole pairs differed by $1 \%$ as a function of symbolic distance, but unrelated pairs differed by $7 \%$ as a function of symbolic distance. This interaction between relatedness and symbolic distance was highly reliable $\left[F(1,20)=14.8, M S_{\mathrm{e}}=0.0009, p<\right.$ $.001]$. In addition, fewer errors were made on related pairs $(M=.04)$ than on unrelated pairs $[M=.07, F(1,20)=$ $\left.61.5, M S_{\mathrm{e}}=0.0005, p<.001\right]$, and fewer errors were made on far pairs $(M=.03)$ than on close pairs $[M=.08$, $\left.F(1,20)=58.4, M S_{\mathrm{e}}=0.0006, p<.001\right]$. Taken together the RT and error data indicate that the part-whole relation greatly reduces the role of symbolic distance in the judgment process.

\section{EXPERIMENT 2}

According to most models of comparative judgment, we judge that horses are larger than dogs by comparing the sizes of the two animals rather than by retrieving from memory the proposition "horses are larger than dogs." $\mathrm{Al}$ though the data from Experiment 1 can be explained by the facilitation hypothesis, the fact that part-whole relations attenuated the effect of symbolic distance raises the possibility that this relation provides a direct source of relational information.

The following experiment examines the effect of relatedness on the comparison of magnitude information by requiring participants to judge differences in the magnitudes of objects. More specifically, participants judged which of two objects was closer in size to a third, reference, object.

In a reference point task (Holyoak, 1978), participants are required to judge whether the difference in magnitude

Table 2

Mean Reaction Times (RTs) and Percentage Error (PE) for Part-Whole Pairs and Unrelated Pairs as a Function of Symbolic Distance in Experiment 1

\begin{tabular}{|c|c|c|c|c|c|}
\hline \multirow[b]{3}{*}{ Relatedness } & \multicolumn{4}{|c|}{ Symbolic Distance } & \multirow[b]{3}{*}{ Distance Effec } \\
\hline & \multicolumn{2}{|c|}{ High } & \multicolumn{2}{|c|}{ Low } & \\
\hline & RT & $\overline{\mathrm{PE}}$ & RT & $\mathrm{PE}$ & \\
\hline Part-Whole & 1,519 & 3 & 1,656 & 4 & 137 \\
\hline Unrelated & 1,681 & 4 & 2,020 & 11 & 339 \\
\hline Facilitation & \multicolumn{2}{|c|}{162} & \multicolumn{2}{|c|}{364} & \\
\hline
\end{tabular}


between Object $A$ and a reference object is smaller than the corresponding difference between another Object B and the same reference object. These judgments are similar to comparative judgments because they are sensitive to differences in the magnitudes of the objects that are being compared. As the differences between the reference object and the two alternatives become more discrepant, the judgment becomes easier. For example, it is easier for participants to determine that San Francisco is further from Philadelphia than it is from Salt Lake City than it would be for them to determine that the same distance between San Francisco and Philadelphia is smaller than the distance from San Francisco to New York City (Holyoak \& Mah, 1982).

The advantage of the reference point task in determining the viability of the facilitation hypothesis is that it is possible to construct triples in which the magnitude of the referent object falls between the magnitudes of the two objects to which it is being compared. Because the alternatives fall on each side of the reference object, the order of the alternatives on the dimension cannot be used to determine which of the two alternatives is closer to the referent object. Consider the triple BIRD, cat, egg, where the referent object is capitalized and cat and egg are the two alternatives. Even though one might know the ordering of cat, BIRD, and egg, this information cannot be used to determine whether BIRD is closer in size to a cat or to an egg. Instead, the correct answer must be determined by comparing the difference in the magnitudes of cat and BIRD with the same difference for BIRD and egg and selecting the smaller difference.

This difference between reference point judgments and standard comparative judgments has implications for the predictions that the two hypotheses make about the effects of relatedness on reference point judgments. According to standard models of comparative judgment, processing should be quite similar in the two tasks because both tasks require the retrieval of magnitude information from long-term memory followed by an evaluation of magnitude differences. ${ }^{1}$ Thus, if the facilitation hypothesis is correct, the inclusion of a part-whole pair in these triples should facilitate judgments and reduce the effects of the difference in the magnitude differences between each alternative and the referent object.

In contrast, the direct retrieval hypothesis claims that the advantage of part-whole pairs in a comparative judgment task is produced by the increased availability of stored relational information. By eliminating the benefit of ordinal information for certain triples, the reference point task eliminates this potential advantage for these triples when they have embedded part-whole pairs. If the effect of including a part-whole relation in a triple is quite comparable to the effect of the part-whole relation on comparative judgments, the direct retrieval hypothesis is probably incorrect because strictly ordinal information cannot be used to solve these triples. Thus, a reference point task in principle can isolate the evaluation of magnitude information from uses of stored relational information.

\section{Method}

Materials. Triples were constructed from 14 part-whole pairs selected from the same pool of items that was used in Experiment 1. One member of each pair served as the referent and the other member served as an alternative. A third item that was unrelated to either member of the part-whole pair was selected as the second alternative. Each of these 14 part-whole relations was used to construct two kinds of triples. In the close set of triples the difference between the rated size of the closer alternative and the referent was just slightly smaller than the comparable difference between the further alternative and the referent, but in the far set of triples this discrepancy between these two differences was greater. A control triple was constructed for each of these 28 triples by replacing the referent with an unrelated object of identical rated magnitude. Thus, there were 28 triples and their controls for a total of 56 triples.

The difference in the difference between the rated sizes of the two alternatives and the referent was on average 5.39 scalar units for the far triples and 1.04 scalar units for the close triples. The difference in the ratio of the magnitude of each object to the magnitude of the referent was .40 scalar units for the far triples and .19 scalar units for the close triples. Thus, if participants assessed the magnitude difference between an alternative and the referent by using a subtraction operation or by using a ratio operation, the difference in the subjective magnitudes of the constituents of far triples should have been greater than the comparable differences in the constituents of close triples.

Design. Triples varied in terms of the relative difference in the magnitude of each alternative and the referent and whether or not one of these alternatives formed a part-whole pair with the referent. The two alternatives were presented prior to the presentation of the referent to minimize the effects of reading time.

Each triple was presented twice within a block, with the two alternatives appearing once to each side of the screen. The ordering of stimuli within a block was randomly determined for each participant with the constraint that a triple with the same part-whole relation or its control could not appear again within two trials. Thus, each triple appeared six times during the course of the experiment for a total of 336 trials.

Procedure. Participants were run on individual computers with VGA monitors. Each trial began with a ready signal (the word ready) that remained on the screen until the participant pressed the space bar. This barpress initiated the presentation of the two alternatives in lowercase letters. After $500 \mathrm{msec}$, the referent appeared above the two alternatives in uppercase letters. Participants were told to choose the target object that was closer in size to the referent object. Participants indicated their choice by pressing the key labeled " $j$ " with their right hand if the correct item was on the right or by pressing the key labeled " $f$ " with their left hand if the correct item was on the left. RT was measured from the onset of the two alternatives. If the participant made an incorrect response, the message "WRONG ANSWER" appeared for $7 \mathrm{sec}$. If a participant responded correctly but took longer than $7 \mathrm{sec}$ to respond, the message "TOO SLOW" appeared on the screen.

As in Experiment 1, participants were told about both their accuracy and their mean response latency at the end of each block. They were again strongly encouraged to respond accurately on at least $95 \%$ of their trials and to respond as quickly as possible while maintaining this level of accuracy. The first 16 trials were practice trials with materials not among the test stimuli.

Participants. Forty-six undergraduates at Barnard College received $\$ 8$ for their participation. All participants were native English speakers and met an error criterion of 65 errors $(19 \%)$. 
Table 3

Reaction Times (RTs, in Milliseconds) and Percentage Error (PE) for Related and Unrelated Triples as a Function of Magnitude Difference in Experiment 2

\begin{tabular}{|c|c|c|c|c|c|}
\hline \multirow[b]{3}{*}{ Relatedness } & \multicolumn{4}{|c|}{ Magnitude Difference } & \multirow[b]{3}{*}{ Distance Effect } \\
\hline & \multicolumn{2}{|c|}{ Far } & \multicolumn{2}{|c|}{ Close } & \\
\hline & RT & $\mathrm{PE}$ & $\mathrm{RT}$ & $\mathrm{PE}$ & \\
\hline Part-Whole & 2,495 & 6 & 2,661 & 13 & 166 \\
\hline Unrelated & 2,630 & 7 & 2,744 & 10 & 114 \\
\hline Facilitation & \multicolumn{2}{|c|}{135} & \multicolumn{2}{|c|}{83} & \\
\hline
\end{tabular}

\section{Results}

The results appear in Table 3. Mean RTs and error rates are given separately for each level of magnitude difference and relatedness. Overall error rates were moderate $(9 \%)$ and correlated positively with RT (.63). Any RT more than 3.5 SDs from the mean RT of a participant was excluded from further analysis.

An initial inspection of Table 3 indicates that related triples were faster than their controls that did not contain a part-whole relation $\left[F(1,45)=36.5, M S_{\mathrm{e}}=14,965\right.$, $p<.001]$ and that judgments of far triples were faster than judgments of close triples $\left[F(1,45)=61.03, M S_{\mathrm{e}}=\right.$ $14,735.6, p<.001]$. Although the advantage of related triples appears to support the facilitation hypothesis over the direct retrieval hypothesis because the facilitation hypothesis predicts that related triples should be faster to process than their controls, the exact pattern of results is quite different from the pattern that was observed for standard comparative judgments in Experiment 1. In contrast to the fact that relatedness had a greater impact on close than far judgments in the comparative judgment task, the advantage of related triples was greater for far triples $(M=135 \mathrm{msec})$ than it was for close triples [ $M=$ $\left.83 \mathrm{msec}, F(1,45)=4.48, M S_{\mathrm{e}}=7,087.3, p<.05\right]$. Thus, the direction of the interaction between distance and relatedness that is observed in a standard comparative judgment task was reversed in this task.

In general the error data support the conclusions drawn from the RT data, even though they differ from the RT data in several respects. The main difference between the RT data and the error data is that performance in the related close condition (.13) was noticeably worse than in the unrelated close condition (.10) even though the related far pairs (.06) exhibited a slight advantage over their controls $\left[.07, F(1,45)=24.8, M S_{\mathrm{e}}=0.0008, p<\right.$ $.001]$. This difference for close pairs was large enough to produce a small decrement in accuracy of related triples $\left[F(1,45)=6.5, M S_{\mathrm{e}}=0.0008, p<.05\right]$. Finally, performance on far triples (.06) was more accurate than on close triples $\left[.12, F(1,45)=63.6, M S_{\mathrm{e}}=0.0024, p<.001\right]$. Although the error data do not match the RT data exactly, it is important to point out that the magnitude of the interaction between relatedness and symbolic distance that was observed in the RT data would probably have been greater if participants had been trading speed for accuracy across conditions because RT would have in- creased in the close related condition and this increase would have decreased the size of the relatedness effect for close triples relative to far triples.

\section{Discussion}

The smaller 83-msec effect of relatedness on RT for close triples is important because it is incompatible with either of the two hypotheses about the effects of relatedness on comparative judgment that were outlined in the introduction. Consistent with previous research (Holyoak, 1978; Holyoak \& Mah, 1982; Shoben, Çech, \& Schwanenflugel, 1983), the present results demonstrate that judgment difficulty increases as the two distances between each alternative and the referent become more similar within a triple. Although the general advantage of related triples is consistent with the facilitation hypothesis, the form of the interaction between symbolic distance and relatedness is inconsistent with the facilitation hypothesis. According to the facilitation hypothesis, close triples are harder to answer than far triples because more repetitive searches are required to determine which distance between each alternative and the referent is larger. If relatedness speeds retrieval of magnitude information, the effect of relatedness should have been larger for close triples than for far triples because differences due to the speed with which magnitude information can be retrieved for related and unrelated items should be magnified as the number of searches increases. Instead of attenuating this effect of the difference in the distance between each alternative and the referent, the part-whole relation actually produced a larger effect of distance in related triples than in unrelated controls. The fact that the effect of relatedness diminished in the more difficult close triples indicates that relatedness did not facilitate an iterative search of memory.

The initial version of the direct retrieval hypothesis is clearly incorrect because strictly ordinal information cannot be used to solve the bilateral triples that were used in this experiment. However, it is possible that partwhole pairs provide very coarse grained information about the differences in the magnitude of their constituents that could be used to solve some triples. For example, if the fact that "a toe is much smaller than a foot" was explicitly represented in memory, it might be faster to retrieve this fact than to compute the magnitude difference when trying to determine if a foot was closer in size to a toe or to a teapot.

This coarse-grained information can be distinguished from strict ordinal relations in that it is possible to distinguish among several levels of magnitude difference. For example, one might recognize a car as being very much larger than a headlight, much larger than a door, and somewhat larger than a chassis. By this account, the part-whole relation provides more information about the differences between members of a pair than is provided by an ordinal-level scale, but it provides less information about these differences than is provided by an intervallevel scale because it is not possible to order the differ- 
ences between pairs whose members stand in the same relation to each other on the basis of this information. For example, retrieving the fact that "a tree is much larger than a branch" would not distinguish this pair from the pair branch-leaf if a branch was also represented as being "much larger" than a leaf.

The utility of this information in judgments about triples depends on the similarity of the differences between each alternative and the referent. If the two alternatives are comparable distances from the referent, it is unlikely that coarse-grained information can distinguish the two distances. For example, the part-whole relation would not help to determine whether a branch was closer in size to a tree or to a leaf if a tree was represented as standing in the same relation to a branch as a branch was to a leaf. However, as the discrepancy in the distance between each alternative and the referent increased, these stored facts would become more useful. For example, the judgment that a tree was "much larger" than a leaf could be used to determine that this difference was greater than the difference between a leaf and a penny if a leaf was judged to be only "larger" than a penny. By this reasoning, one would expect to find a greater advantage for far related triples than close related triples because coarsegrained information about magnitude differences would be more likely to differ when the comparison difficulty of triples was relatively easy.

In contrast to judgments of triples, standard comparative judgments only require ordinal-level information. Given that ordinal-level information is sufficient to solve a standard comparative judgment, the fact that a "tree is much larger than a leaf" would not be any more or less helpful than the fact that a "tree is larger than a branch" because either distinction is sufficient to solve a standard comparative judgment. Thus, the advantage of part-whole pairs in a standard comparative judgment task can be explained if it is assumed that this information can be directly retrieved and used on some portion of judgments.

\section{EXPERIMENT 3}

The form of the interaction between relatedness and comparison difficulty in Experiment 2 was a striking and to date novel departure from the results of several studies of comparative judgments that have manipulated either category membership or semantic association (Holyoak et al., 1979; Kosslyn et al., 1977; Maki, 1981; Pliske \& Smith, 1979; Sailor \& Shoben, 1993, 1996). This reversal of the interaction between comparison difficulty and relatedness in the reference point task is important because it suggests that neither the facilitation hypothesis nor the original version of the direct retrieval hypothesis is correct.

Experiment 3 was designed to provide an even more direct contrast between a task that can be solved using ordinal information and a task that requires participants to judge differences in the magnitudes of objects than was provided by the previous experiments. Although the reference point task that was used in Experiment 2 provided a relatively direct contrast to the standard comparative judgment task, there were several potentially significant differences in the materials that were used in the first two tasks. First, the related pairs in Experiment 1 were composed of entirely related items (e.g., a partwhole pair), but the related triples were constructed from a part-whole pair (e.g., the related alternative and the referent) and an unrelated pair (e.g., the unrelated alternative and the referent). This difference meant that a part-whole relation could be used to solve a part-whole pair in the comparative judgment task but at least one pair in every related triple could not be evaluated by using the part-whole relation. A second difference between the materials from Experiments 1 and 2 was that some of the specific pairs that were used to construct the items differed across the two experiments.

The goal of the present experiment was to eliminate these factors as possible explanations of the different form of the interaction between relatedness and comparison difficulty in Experiments 1 and 2. Toward this end, participants made two kinds of judgments. First, they determined which of two differences in magnitude was greater. These judgments required participants to compare the distance between the members of one pair with the distance between the two members of a second pair. They were similar to judgments of triples because they cannot be solved by knowing the ordering of each pair but must be solved by determining the difference in the sizes of the two members of each pair (Birnbaum, Anderson, \& Hynan, 1989; Petrusic, Baranski, \& Kennedy, 1998). Comparison difficulty was manipulated by varying the differences in the size of the members of one pair relative to the differences in the sizes of a second pair. On half of the trials, both pairs were related and on the other half of the trials both pairs were unrelated. We will refer to these judgments of two pairs as comparisons of pairs and the two pairs as quads (Petrusic et al., 1998) because they involve an evaluation of four terms.

After participants performed these judgments, they performed comparative judgments on the individual pairs that were used in combination to form each quad in the first part of the experiment. According to the modified direct retrieval hypothesis, the part-whole relation provides coarse-grained information about a limited number of different levels of magnitude difference between the two members of a related pair (e.g., "much smaller," "smaller," "larger," "much larger," etc.). This account claims that relatedness facilitated close pairs more than far pairs in the comparative judgment task because the ability to use this information to order a pair's members became more useful as the difficulty of computing relative magnitude from differences in absolute magnitude increased. In contrast, this account claims that this coarsegrained information was more likely to be useful in making judgments about far triples than close triples because the discrepancy between the distance of each alternative to the reference object may have been too small to be re- 
solved by this coarse-grained information in the case of close triples. In the present experiment, any difference in the results between the comparative judgment task and the comparison of pairs task cannot be attributed to a difference in materials because the quads were constructed from the pairs that were used in the comparative judgment task. In addition, any difference in the effects of relatedness on judgment difficulty in the two tasks cannot be attributed to differences in the availability of part-whole relations for the materials in the two tasks because both pairs in the related quads are related. Therefore, if the comparative judgments replicate the results of Experiment 1 and the judgments of quads replicate the results of Experiment 2 , the combined results would eliminate these alternative accounts and further support the conclusion that the kind of information being derived from the part-whole relation in the reference point and comparison of pairs tasks is different from the kind of information that is used in a standard comparative judgment.

\section{Method}

Materials. Quads were constructed from 22 part-whole pairs selected from the same pool of items that was used in Experiments 1 and 2. Related quads were constructed by pairing each pair with one of the other part-whole pairs. Far quads were constructed by pairing a pair whose member's magnitudes differed greatly with a pair whose members did not differ greatly (e.g., door-keyhole vs. fireplace-mantel). Close quads were created by re-pairing these same pairs so that the discrepancy in the magnitudes of the constituent pairs was smaller (e.g., car-headlight vs. door-keyhole). Control quads were constructed by switching one member of each pair in the quad with a member of a pair in a different quad whose rated magnitude was comparable. Thus, there were 11 quads in each of four conditions for a total of 44 quads. The difference in the difference between the rated sizes of each pair member was 3.66 scalar units for the far related quads, 1.57 scalar units for the related close quads, 3.65 scalar units for the unrelated far quads, and 1.5 scalar units for the unrelated close quads. The ratios of the distance between the members of the closer pair and the farther pair were $.357, .603$, .368 , and .631 for quads in the far related, close related, far unrelated, and close unrelated conditions, respectively.

The pairs that were used for the comparative judgment task at the end of the experiment were simply the individual pairs that were used to form the quads. Although it was not possible to find a set of materials that yielded an equal number of pairs in all four conditions and that simultaneously satisfied the requirements of the comparison of pairs task, a very close solution was obtained by dividing these pairs into 21 far pairs whose magnitude difference exceeded 3.85 scalar units and 23 close pairs whose magnitude difference was less than 3.85 scalar units. Ten of the 21 far pairs were related and 12 of the 23 close pairs were related. The mean magnitude differences for far related, far unrelated, close related, and close unrelated were $6.21,6.02,2.36$, and 2.37 scalar units, respectively.

Design. Quads varied in terms of the relative difference in magnitude of each pair and whether the pairs formed a part-whole pair. Within each quad, the larger member of each pair always appeared to the left of the smaller member of that pair (e.g., shoe-heel vs. tree-leaf). This ordering was established to minimize any advantage that part-whole pairs might have in establishing the direction of the difference within each half of the quad. The pairs used in the comparative judgment task varied in terms of their symbolic distance and whether or not their members formed a part-whole pair.

Procedure. In the first phase, participants judged which constituent pair in a quad had a greater difference in the size of its mem- bers, and in the second phase they judged the relative magnitude of the members of a pair. In each phase, stimuli were presented four times for a total of 176 trials. The constituents of each stimulus (i.e., pairs for quads and individual object names for pairs) were presented equally often to the left and right side of the screen. The ordering of stimuli within a phase was randomized for each participant.

Participants were run on individual computers with VGA monitors. Each trial began with a ready signal (the word ready) that remained on the screen until the participant pressed the space bar. This barpress initiated the presentation of a stimulus. In the comparison of pairs task, two dashes separated the members of each pair and the two pairs were separated on the screen by approximately $5 \mathrm{~cm}$. In both tasks, participants indicated their choice by pressing the key labeled " $\mathrm{j}$ " with their right hand if the correct constituent was on the right or by pressing the key labeled " $f$ " with their left hand if the correct constituent was on the left. RT was measured from the onset of the stimulus. If the participant made an incorrect response, the message "WRONG ANSWER" appeared for $4 \mathrm{sec}$.

In the comparison of pairs task, participants were instructed to judge the similarity of two objects only in terms of their size. To ensure that participants were judging the similarity of the sizes of two objects and not their similarity in other respects, an example was given in which the size of an object (e.g., cat) was contrasted with the size of an object that was similar in other respects but differed greatly in size (e.g., lion) and an object that was quite similar in size even though it differed substantially in other respects (e.g., shoebox).

As in previous experiments, participants were told about both their accuracy and their mean response latency at the end of each block. They were strongly encouraged to respond accurately on at least $85 \%$ of their trials and to respond as quickly as possible while maintaining this level of accuracy. The first eight trials in each phase of the experiment were practice trials with materials not among the test stimuli.

Participants. Twenty-eight undergraduates at Barnard College received $\$ 8$ for their participation. Two of these participants were excluded from further analyses for failing to meet an error criterion of 36 errors $(20 \%)$ in the comparison of pairs task.

\section{Results}

Comparison of pairs. The results appear in Table 4. Mean RTs, and error rates are given separately for each level of comparison difficulty and relatedness. Overall error rates were moderate $(8 \%)$ and correlated positively with RT (.501). Any RT more than $3.5 S D$ s from the mean RT of a participant was excluded from further analysis. The data for one quad in the close related condition and two quads in the close unrelated condition were eliminated because participants made more than $50 \%$ errors on these items. RT and error data were subjected to a two-way repeated measures analysis of variance (ANOVA) with distance (far vs. close quads) and relatedness (part-whole vs. unrelated quads) serving as within-subjects factors.

Table 4

Reaction Times (RTs, in Milliseconds) and Percentage Error (PE) for Part-Whole Pairs and Unrelated Pairs as a Function of Magnitude Difference for Quad Judgments in Experiment 3

\begin{tabular}{|c|c|c|c|c|c|}
\hline \multirow[b]{3}{*}{ Relatedness } & \multicolumn{4}{|c|}{ Symbolic Distance } & \multirow[b]{3}{*}{ Distance Effect } \\
\hline & \multicolumn{2}{|c|}{ Far } & \multicolumn{2}{|c|}{ Close } & \\
\hline & RT & $\mathrm{PE}$ & RT & $\mathrm{PE}$ & \\
\hline Part-Whole & 3,627 & 7 & 4,042 & 10 & 415 \\
\hline Unrelated & 4,216 & 8 & 4,243 & 11 & 27 \\
\hline Facilitation & \multicolumn{2}{|c|}{589} & \multicolumn{2}{|c|}{201} & \\
\hline
\end{tabular}


In general, the results of this task are quite consistent with the results of the reference point task in Experiment 2. RT decreased as a function of both magnitude difference $\left[F(1,25)=16.7, M S_{\mathrm{e}}=76,282, p<.001\right]$ and relatedness $\left[F(1,25)=27.3, M S_{\mathrm{e}}=148,846, p<.001\right]$. More importantly, the effect of relatedness was greater for far quads than it was for close quads. Related far pairs were $589 \mathrm{msec}$ faster than unrelated far pairs, whereas related close pairs were only $201 \mathrm{msec}$ faster than unrelated close pairs $\left[F(1,25)=15.4, M S_{\mathrm{e}}=63,145, p<.001\right]$.

The error data were generally consistent with the RTs. Participants made more errors on close quads than far quads $\left[F(1,25)=8.2, M S_{\mathrm{e}}=0.0044, p<.01\right]$. Although participants made slightly more errors on unrelated than related quads, neither this difference nor the interaction between magnitude difference and relatedness was significant $(F<1$ for both).

Comparative judgments. The results appear in Table 5. Again, RTs that exceeded 3.5 SDs from a participant's mean RT were excluded from further analysis. In contrast to the judgments of quads, these data are quite consistent with the results of the comparative judgment task in Experiment 1. RT decreased as a function of both magnitude difference $\left[F(1,25)=117.4, M S_{\mathrm{e}}=\right.$ $12,387, p<.001]$ and relatedness $\left[F(1,25)=63.9, M S_{\mathrm{e}}=\right.$ $13,748, p<.001]$. Most importantly, the form of the interaction between distance and relatedness paralleled the form obtained for comparative judgments in Experiment 1 in that the effect of relatedness was much greater for close pairs than it was for far pairs. Related close pairs were $239 \mathrm{msec}$ faster than their controls, whereas related far pairs were only $145 \mathrm{msec}$ faster than their controls. This difference was reliable $\left[F(1,25)=7.3, M S_{\mathrm{e}}=\right.$ $7,788, p<.05]$.

The error data exhibited an identical pattern of effects. Participants made fewer errors on far pairs $(M=.01)$ than on close pairs $\left[M=.04, F(1,25)=53.3, M S_{\mathrm{e}}=0.0051\right.$, $p<.001]$, and they made fewer errors on part-whole pairs $(M=.02)$ than on unrelated pairs $[M=.03, F(1,25)=$ 7.6, $\left.M S_{\mathrm{e}}=0.0005, p<.05\right]$. Similar to the RT analyses, the advantage of part-whole pairs over their controls was greater for close pairs than it was for far pairs $[F(1,25)=$ 26.6, $\left.M S_{\mathrm{e}}=0.0004, p<.001\right]$. In fact, participants made marginally fewer errors on unrelated far pairs than on related far pairs, but about $3 \%$ more errors on related close pairs than on unrelated close pairs.

\section{Discussion}

The significance of this replication of the earlier findings is that it eliminates several artifactual accounts of the results of the reference point and comparison of pairs tasks. The same 44 pairs were used to construct both far and close judgments in the comparison of pairs task. This fact makes it difficult to argue that the larger magnitude difference effect for related quads occurred because of some uncontrolled difference in the pairs that were used to construct quads in the two conditions. The results of the comparative judgment task add to this dif- ficulty because they demonstrate that the presence of the part-whole relation in these materials was sufficient to produce an attenuation of the symbolic distance effect in a standard comparative judgment task.

In the introduction, we noted that related pairs could have been solved in Experiment 1 by retrieving relative magnitude but that at least one magnitude difference had to be computed for the related triples in Experiment 2 because one of the two alternatives was not related to the referent. The present results suggest that this difference was not responsible for the reversal of the interaction between relatedness and comparison difficulty that was observed in the reference point task. If it had been responsible for this reversal, then the use of two part-whole pairs to construct quads should have produced a greater effect of the part-whole relation in the close quads than in the far quads.

Notably, the most significant difference between the results of the comparison of pairs task and the reference point task is that the amount of facilitation provided by the part-whole relation was larger in this experiment than it was in the reference point task. This observation appears to be true whether facilitation is calculated as a difference between related and unrelated RT or whether related RT is calculated as a percentage of unrelated RT in the three tasks. For example, the decrease in RT for related materials was only $4 \%$ of unrelated RT in the reference point task. In contrast, this decrease was approximately $9 \%$ in the comparison of pairs task.

This difference in facilitation suggests that the presence of an unrelated pair in the related triples may have reduced the amount of facilitation that was recorded in this task relative to the comparison of pairs task. According to the modified direct retrieval account, judgments of related triples or quads are facilitated to the extent that it is faster to solve them by retrieving coarse-grained information about magnitude differences than by computing these differences from the absolute magnitudes of individual items. In the case of quads, this coarse-grained information was potentially available for both pairs because both were related. In the case of the related triples, this coarse-grained information was theoretically available to determine the magnitude difference between the referent and the related alternative but not available between the referent and the unrelated alternative. This difference means that the advantage of the related triples

Table 5

Reaction Times (RTs, in Milliseconds) and Percentage Error (PE) for Part-Whole Pairs and Unrelated Pairs as a Function of Symbolic Distance for Comparative Judgments in Experiment 3

\begin{tabular}{|c|c|c|c|c|c|}
\hline \multirow[b]{3}{*}{ Relatedness } & \multicolumn{4}{|c|}{ Symbolic Distance } & \multirow[b]{3}{*}{ Distance Effect } \\
\hline & \multicolumn{2}{|c|}{ Far } & \multicolumn{2}{|c|}{ Close } & \\
\hline & RT & PE & RT & $\overline{\mathrm{PE}}$ & \\
\hline Part-Whole & 1,370 & 1 & 1,560 & 3 & 190 \\
\hline Unrelated & 1,515 & 0 & 1,799 & 6 & 284 \\
\hline Facilitation & \multicolumn{2}{|c|}{145} & \multicolumn{2}{|c|}{239} & \\
\hline
\end{tabular}


over their unrelated controls should have been smaller than the advantage of the related quads over their unrelated controls. Admittedly it is difficult to compare performance across two tasks with very different mean RT, but the fact that facilitation is greater in the comparison of pairs task than in the reference point task is entirely consistent with the modified direct retrieval hypothesis.

\section{GENERAL DISCUSSION}

In the introduction, we outlined two contrasting accounts of how part-whole relations might facilitate standard comparative judgments: the facilitation hypothesis and the direct retrieval hypothesis. Although each of these explanations can account for aspects of our data, neither hypothesis can account for the combined set of results without further modification.

According to the facilitation hypothesis, we can determine the relative size of a part-whole pair more quickly because the association between the terms facilitates the retrieval of absolute magnitude information from memory. This facilitation is strongest when the difference in the magnitudes of two items is small because a more extensive memory search must be conducted before relative magnitude can be successfully determined from absolute magnitude. The results of the reference point and the comparison of pairs task are inconsistent with the facilitation hypothesis because the part-whole relation had a larger effect when magnitude differences were great than when they were small.

According to the direct retrieval hypothesis, it is easier to determine the relative magnitude of the members of a part-whole pair because ordinal information is frequently available for these pairs and it can be used to determine their relative magnitude instead of computing their relative magnitude from information about their absolute magnitudes. The problem for this hypothesis is that the reference point task and comparison of pairs task are more easily performed for materials constructed from part-whole pairs than for materials constructed from unrelated pairs. This result is incompatible with the original version of the direct retrieval hypothesis because ordinal information should not be useful for comparing the magnitudes of two differences.

Although this pattern is not compatible with the original direct retrieval hypothesis, a modified version of the direct retrieval hypothesis can account for it. This modification includes the possibility that information can be retrieved about part-whole pairs that can be used to order some but not all pairwise differences among part-whole pairs. According to this modified account, very coarsegrained information about the general level of the magnitude differences of part-whole pairs is available. This information takes the form of several different relations that represent the order of a pair on a dimension and a general level of magnitude difference such as "much larger," "larger," "smaller," "much smaller," and so on. In a standard comparative judgment task, each of these relations is sufficient to determine the relative magnitude of a pair of items. As was proposed in the original direct retrieval hypothesis, this information is most useful when computing relative magnitude is time-consuming. Therefore, the part-whole relation facilitates comparisons of close pairs more than far pairs.

In contrast to the standard comparative judgment task, the reference point and comparison of pairs tasks require that participants determine which of two magnitude differences is greater. Coarse-grained distinctions about magnitude differences are sufficient only when the general magnitude difference coded by one of these relations is different from the magnitude difference to which it is being compared. For example, in the comparison of pairs task, the decision that the difference between a car and a headlight is bigger than the difference between a bed and a mattress is facilitated because the differences between the two pairs would be labeled differently. In contrast, when the differences between the members of two pairs are more similar, the information is not useful because it may very well be the same. For example, it is probably hard to distinguish the difference between a car and a headlight from the difference between a tree and a leaf.

The viability of the modified direct retrieval account depends substantially on the plausibility of the idea that relational information of this sort is explicitly represented in memory. Previous accounts of comparative judgment rejected the idea that relational information was explicitly represented in part because of the memory demands of this relational coding scheme. However, the significance of these memory demands was predicated on the view that most comparisons were solved by directly retrieving relative magnitude. In the present experiments, judgments were limited to related pairs that were judged to be good examples of the part-whole relation. In the Holyoak et al. (1979) study, the associated items were judged to be strongly associated in a norming experiment. Clearly, the memory demands are substantially reduced if relational information is represented for a relatively limited subset of items. This analysis suggests that relational coding is limited to strongly associated pairs or pairs that enter into a relation that is correlated with their ordering on a dimension. For example, precise temporal differences may not be terribly critical for determining that more time is required to change a flat than to remove a hubcap because removing a hubcap is part of the scripted activities organized under the general routine of changing a flat.

Contemporary theories of the comparative judgment process have focused exclusively on processes by which relative magnitude is computed from absolute magnitude. The present results are consistent with other work (Kosslyn et al., 1977; Maki, 1981; Pliske \& Smith, 1979; Sailor \& Shoben, 1993, 1996), which suggests that these theories must be modified by the addition of other processes that do not utilize differences in absolute magnitude to determine relative magnitude. In the case of the part-whole relation, we have argued that this process in- 
volves the direct retrieval of relative magnitude from memory. In other cases, this process may depend on retrieving or generating discrete magnitude categories (Kosslyn et al., 1977; Maki, 1981; Pliske \& Smith, 1979; Sailor \& Shoben, 1993, 1996). For example, participants may code all animals as small and all buildings as large if this distinction is perfectly correlated with relative magnitude within the experiment (Sailor \& Shoben, 1993). The attenuation of symbolic distance for related materials within these studies can be explained by assuming that relative magnitude is determined by a race between two independent sets of processes. One of these sets of processes computes relative magnitude from stored absolute magnitude information. These processes are assumed to operate along the lines that have been specified within traditional accounts of comparative judgment. The other set of processes determines relative magnitude by exploiting a semantic relation that is correlated with relative magnitude. In the case of the part-whole relation, it is assumed that this operation involves retrieving stored relational information about the pair. Because these two sets of processes are assumed to be independent, factors that affect the computation of relative magnitude from stored magnitude information will not, in general, affect the duration of the processes that retrieve stored relational information. At the same time, factors that affect the retrieval of stored relational information will not generally affect the duration of processes that compute relative magnitude information. The factors that are presumed to affect the duration of the processes used to compute relative magnitude from absolute magnitude are primarily those factors that have already been identified in the literature on comparative judgment: symbolic distance, serial position, and congruity. Although no completely satisfactory model exists regarding exactly how these factors affect the computation of relative magnitude, the effects of these variables are well known. Each of these factors will affect judgment by affecting the duration of these processes. Judgments will therefore be faster and more accurate when symbolic distance is great, the items are of extreme magnitude, and the items are congruent with the instructions. Of course, the impact of these variables is presumed to be mitigated by the action of the retrieval processes. More specifically, the magnitude of the effects of symbolic distance, serial position, or congruity will be much smaller when the retrieval processes are more likely to determine the outcome of the judgment process than when the outcome is likely to be determined by the computational processes.

In summary, a major contribution of this paper is to establish that the facilitation hypothesis cannot account for the effect of the part-whole relation on judgments of triples or quads. As we noted in the initial presentation of the direct retrieval hypothesis, a similar test cannot be performed directly on comparative judgments because they do not logically require judgments of the magnitude differences. However, the present results indicate that retrieving and comparing information about the absolute magnitudes of objects is not facilitated by a part-whole relation. For this reason, they are inconsistent with previous explanations of the comparative judgment process, which proposed that relative magnitude is computed on the basis of retrieving absolute magnitude information about the members of a pair (Banks, 1977; Holyoak, 1978; Holyoak \& Mah, 1982). Previous research demonstrated that several factors attenuate the symbolic distance effect. Some of these accounts emphasized the use of discrete magnitude categories (Kosslyn et al., 1977; Shoben \& Wilson, 1997), while others proposed the possibility that relative magnitude was directly retrieved (Holyoak et al., 1979). In both cases, we argue that it is quite likely that this information is processed in parallel with processes that compute relative magnitude from absolute magnitude information. An interesting implication of this parallel architecture is that the symbolic distance effect is likely to be evident even under circumstances in which relative magnitude can be directly retrieved. Previously, researchers have discounted the role of direct retrieval because of a general belief that the presence of a symbolic distance effect was inconsistent with the direct retrieval of magnitude information. If the account offered in this paper is correct, it is quite likely that the direct retrieval of relative magnitude is more common than has been previously believed.

\section{REFERENCES}

ANDERSON, J. R. (1983). The architecture of cognition. Cambridge, MA: Harvard University Press.

AUdLEY, R. J., \& Wallis, C. P. (1964). Response instructions and the speed of relative judgments. I. Some experiments on brightness discrimination. British Journal of Psychology, 55, 59-73.

BANKS, W. P. (1977). Encoding and processing of symbolic information in comparative judgments. In G. H. Bower (Ed.), The psychology of learning and motivation (Vol. 11, pp. 101-159). New York: Academic Press.

Birnbaum, M. H., Anderson, C. J., \& Hynan, L. G. (1989). Two operations for "ratios" and "differences" of distances on the mental map. Journal of Experimental Psychology: Human Perception \& Performance, 15, 785-796.

Collins, A. M., \& LofTus, E. F. (1975). A spreading-activation theory of semantic processing. Psychological Review, 82, 407-408.

Friedman, A. (1978). Memorial comparisons without the "mind's eye." Journal of Verbal Learning \& Verbal Behavior, 17, 427-444.

Glass, A. L., Holyonk, K. J., \& O’Dell, C. (1974). Production frequency and the verification of quantified statements. Journal of Verbal Learning \& Verbal Behavior, 13, 237-254.

HoLYOAK, K. J. (1978). Comparative judgments with numerical reference points. Cognitive Psychology, 10, 203-243.

HolyoaK, K. J., Dumais, S. T., \& MoyeR, R. S. (1979). Semantic association effects in a mental comparison task. Memory \& Cognition, 7, 303-313.

holyoak, K. J., Glass, A. J., \& MaH, W. A. (1976). Morphological structure and semantic retrieval. Journal of Verbal Learning \& Verbal Behavior, 15, 235-247.

HOL YOAK, K. J., \& MAH, W. A. (1981). Semantic congruity in symbolic comparisons: Evidence against an expectancy hypothesis. Memory \& Cognition, 9, 197-204. 
HolyoAK, K. J., \& MAH, W. A. (1982). Cognitive reference points in judgments of symbolic magnitude. Cognitive Psychology, 14, 328-352.

Holyoak, K. J., \& WalkeR, J. H. (1976). Subjective magnitude information in semantic orderings. Journal of Verbal Learning \& Verbal Behavior, 15, 287-299.

JAmieson, D. G., \& Petrusic, W. M. (1975). Relational judgments with remembered stimuli. Perception \& Psychophysics, 18, 373-378.

Kerst, S. M., \& Howard, J. H., JR. (1977). Mental comparisons for ordered information on abstract and concrete dimensions. Memory \& Cognition, 5, 227-234.

Kosslyn, S. M., Murphy, G. L., Bemesderfer, M. E., \& Feinstein, K. J. (1977). Category and continuum in mental comparisons. Journal of Experimental Psychology: General, 106, 341-375.

LiNK, S. W. (1990). Modeling imageless thought: The relative judgment theory of numerical comparisons. Journal of Mathematical Psychol$o g y, 34,2-41$.

MAKI, R. H. (1981). Categorization and distance effects with spatial linear orders. Journal of Experimental Psychology: Learning, Memory, \& Cognition, 7, 15-32.

MARKs, D. F. (1972). Relative judgment: A phenomenon and a theory. Perception \& Psychophysics, 11, 156-160.

MOYER, R. S. (1973). Comparing objects in memory: Evidence sug gesting an internal psychophysics. Perception \& Psychophysics, 13, 180-184.

Nottenburg, G., \& Shoben, E. J. (1980). Scripts as linear orders. Journal of Experimental Social Psychology, 16, 329-347.

Palvio, A. (1975). Perceptual comparisons through the mind's eye Memory \& Cognition, 3, 635-647.

Petrusic, W. M. (1992). Semantic congruity effects and theories of the comparison process. Journal of Experimental Psychology: Human Perception \& Performance, 18, 962-986.

Petrusic, W. M., Baranski, J. V., \& Kennedy, R. (1998). Similarity comparisons with remembered and perceived magnitudes: Memory psychophysics and fundamental measurement. Memory \& Cognition, 26, 1041-1055.

Pliske R. M., \& Smith, K. H. (1979). Semantic categorization in a linear order problem. Memory \& Cognition, 7, 297-302.
SAILOR, K. M., \& Shoben E. J. (1993). The effects of real world knowledge on comparative judgment. Journal of Experimental Psychology: Learning, Memory, \& Cognition, 19, 1321-1327.

SAILOR, K. M., \& SHOBEN, E. J. (1996). The role of categorical information in processing relational attributes. Memory \& Cognition, 24, 756-765.

SHOBEN, E. J. (1976). The verification of semantic relations in a samedifferent paradigm: An asymmetry in semantic memory. Journal of Verbal Learning \& Verbal Behavior, 15, 365-379.

Shoben, E. J., ÇECh, C. G., \& SchWANEnflugel, P. J. (1983). The role of subtractions and comparisons in comparative judgments involving numerical reference points. Journal of Experimental Psychology: Human Perception \& Performance, 9, 226-241.

Shoben, E. J., Çech, C. G., Schwanenflugel, P. J., \& SaILoR, K. M. (1989). Serial position effects in comparative judgments. Journal of Experimental Psychology: Human Performance \& Perception, 15, 273-286.

ShobeN, E. J., \& Wilson, T. L. (1997). Categorization in judgments of relative magnitude. Journal of Memory \& Language, 36, 94-111.

WIERZBICKA, A. (1972). Semantic primitives. Frankfurt: Athenaeum.

\section{NOTE}

1. Several proposals have been made that describe the standard comparative judgment task as a comparison of each object to an implicit reference point (Holyoak, 1978; Holyoak \& Mah, 1982; Jamieson \& Petrusic, 1975). In fact, Holyoak and Mah (1982) proposed that the chief difference between paired comparisons and judgments of triples was that the reference point was explicit in the latter task. Although reference point models are probably unique in assuming that the judgment process is virtually identical in both tasks, comparative judgment models that assume that the effect of part-whole relatedness is to facilitate the retrieval of magnitude information from long-term memory predict an effect of this variable in a reference point task

(Manuscript received October 27, 1997; revision accepted for publication April 26, 1999.) 